\title{
Fibre links for the WEAVE instrument: the making of
}

\section{Mignot, Shan, Bonifacio, Piercarlo, Fasola, Gilles, Horville, David, Caffau, Elisabetta, et al.}

Shan B. Mignot, Piercarlo Bonifacio, Gilles Fasola, David Horville, Elisabetta Caffau, Stéphane Dorent, Sébastien Croce, Sébastien Blanc, Basile-Thierry Melse, Youssef Younès, Florent Reix, Julien Gaudemard, Gavin Dalton, Ellen Schallig, Ian Lewis, Remko Stuik, Kevin Middleton, Georgia Bishop, Don Carlos Abrams, Scott Trager, J. Alfonso Aguerri, Esperanza Carrasco, Antonella Vallenari, Philippe Laporte, Patrice Barroso, Camille Noûs, "Fibre links for the WEAVE instrument: the making of," Proc. SPIE 11450, Modeling, Systems Engineering, and Project Management for Astronomy IX, 114502F (13 December 2020); doi: 10.1117/12.2562305 


\title{
Fiber links for the WEAVE instrument: the making of
}

\author{
Shan Mignot*a, Piercarlo Bonifacio ${ }^{\mathrm{a}}$, Gilles Fasola ${ }^{\mathrm{a}}$, David Horville ${ }^{\mathrm{a}}$, Elisabetta Caffau ${ }^{\mathrm{a}}$, Stéphane \\ Dorent $^{\mathrm{a}}$, Sébastien Croce ${ }^{\mathrm{a}}$, Sébastien Blanc ${ }^{\mathrm{a}}$, Basile-Thierry Melse ${ }^{\mathrm{a}}$, Youssef Younès ${ }^{\mathrm{a}}$, Florent Reix ${ }^{\mathrm{a}}$, \\ Julien Gaudemard $^{\mathrm{a}}$, Gavin Dalton ${ }^{\mathrm{b}, \mathrm{c}}$, Ellen Schallig ${ }^{\mathrm{d}}$, Ian Lewis ${ }^{\mathrm{b}}$, Remko Stuik ${ }^{\mathrm{d}}$, Kevin Middleton ${ }^{\mathrm{c}}$, \\ Georgia Bishop ${ }^{\mathrm{c}}$, Don Carlos Abrams ${ }^{\mathrm{e}}$, Scott Trager ${ }^{\mathrm{f}}$, J. Alfonso Aguerri ${ }^{\mathrm{g}}$, Esperanza Carrasco ${ }^{\mathrm{h}}$, \\ Antonella Vallenari ${ }^{i}$, Philippe Laporte ${ }^{\mathrm{a}}$, Patrice Barroso ${ }^{\mathrm{a}}$, Camille Noûs ${ }^{\mathrm{j}}$. \\ ${ }^{a}$ GEPI, Observatoire de Paris, Université PSL, CNRS, 5 Place Jules Janssen, 92195 Meudon, France; \\ ${ }^{b}$ Dept. of Physics, Keble Road, University of Oxford, OX1 3RH, UK; ${ }^{c}$ RALSpace, Science and \\ Technology Facilities Council, Rutherford Appleton Laboratory, Harwell Oxford, OX11 OQX, UK; \\ ${ }^{\mathrm{d} N O V A}$ Optical Infrared Instrumentation Group at ASTRON, Oude Hoogeveensedijk 4, 7991 PD, \\ Dwingeloo, The Netherlands; ' Isaac Newton Group, 38700 Santa Cruz de La Palma, Spain; \\ ${ }^{f}$ Kapteyn Instituut, Rijksuniversiteit Groningen, Postbus 800, 9700 AV Groningen, The Netherlands;

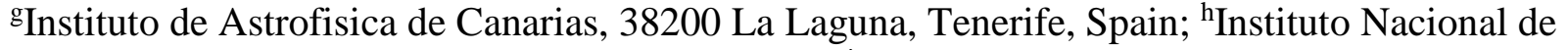

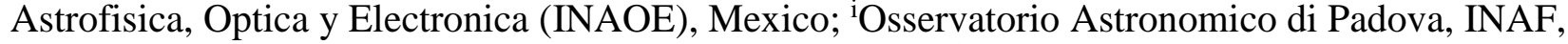 \\ Vicolo Osservatorio 5, 35122, Padova, Italy; ${ }^{j}$ Laboratoire Cogitamus.
}

*shan.mignot@obspm.fr, phone +33 (0) 145077656.

\begin{abstract}
The WEAVE instrument nearing completion for the William Herschel Telescope is a fiber-fed spectrograph operating in three different modes. Two comprise deployable fibers at the prime focus for point-like objects and small integral field units (IFU), the third is a large IFU placed at the center of the field. Three distinct fiber systems support these modes and route the photons to the spectrograph located on the Nasmyth platform 33m away: the first features 960+940 fibers and is duplicated to allow configuring the fibers on one plate while observation is carried out on the other, the second has 20 hexagonal IFUs featuring 37 fibers each, the third is a large array of 609 fibers with twice the former's diameter. The large number of fibers and the diversity of their instantiation have made procurement of the parts and assembly of the custom cables a challenge. They involve project partners in France, the UK and the Netherlands and industrial partners in France, Canada, the USA and China to combine know-how and compress the schedule by parallelizing assembly of the cables. Besides the complex management that this induces, it has called for revising the fibers' handling to relax tolerances and for a rigorous assessment of the conformity of the products. This paper tells the story of the making of the fiber links, presents the overall organization of the procurement and assembly chains together with the inspection and testing allowing for assessing the conformance of the hardware delivered.
\end{abstract}

Keywords: WEAVE, William Herschel Telescope, multi-object spectroscopy, optical fibers, project management, verification, fabrication

\section{INTRODUCTION}

WEAVE is a project for installing a multi-object wide-band dual-resolution spectrograph on the William Herschel Telescope in La Palma, part of the Canary Islands in the North hemisphere. Initiated early in the years 2010, the project has been making significant progress ${ }^{[1-5]}$ and is now nearing completion with first light envisaged for early 2021 . This paper focuses on the fiber optics system and particularly on the 2016-2020 phase during which it was assembled, tested and integrated. While former papers on this system discussed mostly design and the preparation of testing ${ }^{[6-8]}$, this paper tells the real-life story of the making with a view towards lessons learned.

Modeling, Systems Engineering, and Project Management for Astronomy IX, edited by George Z. Angeli,

Philippe Dierickx, Proc. of SPIE Vol. 11450, 114502F · (C) 2020 SPIE

CCC code: $0277-786 \mathrm{X} / 20 / \$ 21 \cdot$ doi: $10.1117 / 12.2562305$ 


\section{FIBER SYSTEM OVERVIEW}

WEAVE is designed as a survey instrument and operates at the prime focus to benefit from a wide field of view ( $2^{\circ}$ in diameter) while reducing the constraints on the field corrector. The spectrograph is installed on the Nasmyth platform and light is transported from the focal plane down the structure of the telescope by optical fibers about $33 \mathrm{~m}$ long. WEAVE supports a variety of science cases including galactic and extra-galactic studies which translate in observing three types of objects differing by the size of the field of view: a multiplicity of point-like objects (MOS), a few extended objects (mIFU) or a single large object (LIFU). The first are not spatially resolved but the other two are via arranging the fibers in integral field units (IFU) of moderate and large sizes. With guide fibers reimaging selected stars for the acquisition and guiding camera, there are hence 4 types of fiber assemblies present on WEAVE. In terms of numbers, the science cases led to $960 \mathrm{MOS}, 20 \mathrm{mIFU}, 1 \mathrm{LIFU}$ and 8 guide fibers.

WEAVE is equipped with a robotic pick-and-place positioner ${ }^{[9,10,11]}$ system allowing for placing the guide, MOS and mIFU fibers at random positions using magnetic buttons on a plate located beyond the focal surface so the objects' light can be picked up by a high index prism ${ }^{1}$ folding the beam into the fibers. Achieving a positioning accuracy of $8 \mu \mathrm{m}$ requires iterations, with a camera placed on the robotic arm imaging the back-illuminated fiber to iteratively correct its position. Even after introducing two robots, positioning the 960 MOS fibers requires an hour's time. Such dead time being clearly unacceptable for exposures lasting an hour on average, this positioning is carried out in parallel to observation at the expense of duplicating the plate, the set of MOS fibers and introducing a mechanism for exchanging plates.

The optimization of the field corrector ${ }^{[12]}$ has led to delivering a non-telecentric $\left(0-4^{\circ}\right.$ over the field of view) $f / 2.75$ beam with a flat focal surface. In order to reduce geometrical focal ratio degradation (FRD), MOS and guide buttons have been designed to tilt the prism and fiber by $2^{\circ}$ while mIFU uses a $92^{\circ}$ prism, both reducing the maximum angles to $\pm 2^{\circ}$. Optimization of the spectrograph has led to its accepting $\mathrm{f} / 3.1$ beams so additional optics had to be introduced at the fiber-level to slow the beam down to f/3.2, a value minimizing intrinsic FRD in the fibers and delivering f/3.1 beams. For MOS fibers, this is carried out via carving a concave spherical lens on the surface of the prism. For mIFU, the field of view being larger, image quality considerations led to separating the lens from the prism.

The case of the LIFU is entirely different as the single extended target allows for having fixed fibers and selecting the target via the telescope's pointing. In this case, the fibers are on-axis eliminating the need for a prism and the focal ratio adaptation is carried out via fore-optics addressing the whole LIFU field of view. While MOS and mIFU fiber cores $(85 \mu \mathrm{m})$ were determined to optimize angular resolution, the LIFU's $(170 \mu \mathrm{m})$ was chosen as a trade-off between the size of the field of view and angular resolution with a view to the complexity of the system, although this led to procuring and managing another type of fiber.

In order to manage and protect such a large and diverse number of fibers, they were assembled as custom cables. The constraints associated to flexibility, resistance to fatigue, resistance to shock and routing from the focal planes to the spectrograph through the positioner and along the telescope structure for MOS, mIFU and LIFU fibers, or more simply to the guide camera for the guide fibers, led to designs featuring from 4 to 6 different sections using ducts made of polyether ether ketone ${ }^{2}$, polyurethane ${ }^{3}$ or polyamide ${ }^{4}$, in addition to the fibers' native polyimide buffer which we took care to preserve throughput the length of the fiber to reduce the risk of damage to the fibers during assembly.

At the spectrograph's end, the fibers are arranged as pseudo-slits to efficiently image the spectra on the detectors. To optimize spectral curvature and focus, the four exchangeable slits corresponding to the two MOS, the mIFU and the LIFU fibers form 3D-curved structures. As a trade-off between optical quality and feasibility, a piece-wise linear approximation to the optimal profile was adopted and the slit split into $40 \times 2,20$ and 27 "slitlets" featuring 24,37 and 21 fibers for MOS, mIFU and LIFU respectively. These slitlets were eventually arrayed parallel to one another and pushed against a field lens ${ }^{[11,13]}$ and, hence, are each cut to a unique angle to best match the profile of the lens. For optimum use

\footnotetext{
${ }^{1} \mathrm{~S}$-TIH 53

2 VICTREX PEEK $381 \mathrm{G}$

${ }^{3}$ Estane 58202 black polyurethane

${ }^{4}$ PMA PMAFLEX POST-12B
} 
of the detector real-estate and reducing cross-talk, the inter-fiber spacing was set to $150 \mu \mathrm{m}$ for MOS and mIFU and $240 \mu \mathrm{m}$ for LIFU (125\% and $110 \%$ of the fibers' outer diameters) with inter-slitlet gaps corresponding to about one fiber. Efficiency optical coupling with the spectrograph also called for controlling the pointing of individual fibers within $0.05^{\circ}$ so very stringent tolerances were placed on the mechanics and assembly of the slitlets.
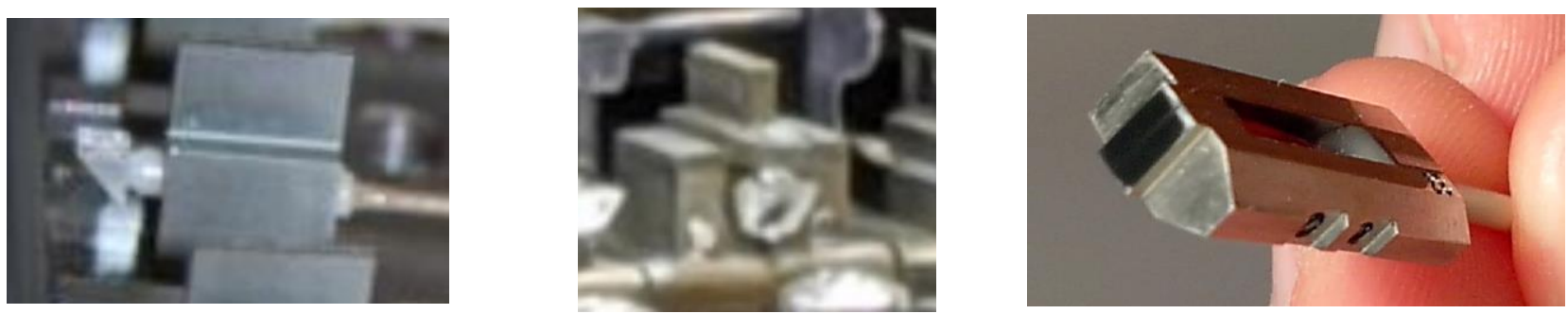

Figure 1. MOS button assembly with its $1.5 \mathrm{~mm}$ prism on the test bench (left), mIFU button assembly with its $2 \mathrm{~mm}$ prism located under the lens holder on its retractor (center) and MOS slitlet during inspection with its 24 fibres (right).

Table 1. Summary of fiber system.

\begin{tabular}{|c|c|c|c|c|}
\hline Parameter & MOS & mIFU & LIFU & guide \\
\hline plate & $A \& B$ & $\mathrm{~B}$ & & $A \& B$ \\
\hline field of view & 1.3" (diameter) & 9" (diameter) & $1.3^{\prime} \times 1.5^{\prime}$ & 5" (diameter) \\
\hline length & $33 \mathrm{~m}$ & $33 \mathrm{~m}$ & $32.25 \mathrm{~m}$ & $3.56 \mathrm{~m}$ \\
\hline number of links & \multirow{2}{*}{$\begin{array}{l}960 \times 2(940 \text { installed } \\
\text { on plate B })\end{array}$} & 20 & 1 & $8 \times 2$ \\
\hline number of fibers & & 740 & 609 (603 installed) & 16 \\
\hline number of cables & $10 \times 2$ & 5 & 1 & $1 \times 2$ \\
\hline number of sections & 6 & 5 & 5 & 4 \\
\hline fiber type & \multicolumn{3}{|c|}{$\begin{array}{c}\text { Polymicro FBP } \\
\text { (step index, broad band, multimode) }\end{array}$} & $\begin{array}{l}\text { Fujikura FIGH-06- } \\
\text { 300PI (multi-core } \\
\text { imaging) }\end{array}$ \\
\hline fiber core diameter & \multicolumn{2}{|c|}{$85 \mu \mathrm{m}$} & $170 \mu \mathrm{m}$ & $270 \mu \mathrm{m}$ \\
\hline optics for injection & lensed prism & lense \& $92^{\circ}$ prism & fore optics & $90^{\circ}$ prism \\
\hline button & $960 \times 22^{\circ}$-slanted & $\begin{array}{l}20\left(2^{\circ} \text {-slanted mount }\right. \\
\text { for lens })\end{array}$ & NA & $8 \times 22^{\circ}$-slanted \\
\hline number of slitlets & $40 \times 2$ & 20 & 27 & NA \\
\hline
\end{tabular}

\section{DEVELOPMENT STRATEGY}

The difficulty of making WEAVE's fiber links was manifold, resulting first from the performance specification (high transmission over the full optical range, low FRD, tiny and ultra-precise opto-mechanics) and increased by the diversity of link types and the numbers - especially the number of individually-addressable fibers for MOS and of fibers in the LIFU IFU.

As a central interface in the system, namely an incarnation of the corrector/spectrograph optical interface dynamically configured by the positioner, the fiber system was developed jointly with the positioner team in the UK notably for the design of the button and with the spectrograph's team in the Netherlands for the design of the optical interfaces and slitlets. The overall dimensions and sectioning of the cable was driven by WEAVE's systems engineer based on inputs from the positioner (robot, retractors, tumbler, structure) for the first $3 \mathrm{~m}$, the telescope (routing along the structure) and the spectrograph (optical bench, slit-exchange mechanism) for the last $3 \mathrm{~m}$.

In terms of fabrication, we decided early to have recourse to industry for the assembly of the cables. Indeed, in spite of their intrinsic diversity, the numbers pointed towards industrialization in order to reliably achieve the desired performance and meet the schedule - besides, such a production effort is not typically part of the scope of a CNRS 
laboratory's activities, as it lacks the appropriate resources in terms of manpower and premises. For maximum benefit, cost-wise and quality-wise, it was then envisaged to have all the assembly work carried out by the same contractor to reduce non-recurrent engineering and have the different products benefit from the others' development. French law requires public laboratories to award such contracts after a call for tender but the selection criteria are defined as part of the call and not limited to minimum cost, making such an approach possible as this contractor could advertise experience and exhibit proofs of abilities. Accordingly, the involvement of industry dates back to PDR (2013), with SEDI-ATI in France delivering prototypes for testing sheathing materials and the MOS design in 2015. GEPI had experience with SEDI-ATI dating from the fabrication of the Medusa fibers for GIRAFFE and the intent was to benefit from their expertise to obtain feedback on the design as regards feasibility and have them develop the processes to prepare the future large-scale production.

One of the aspects making the WEAVE fiber links complex from a project management's point of view is that they are not simply cables. Due to their being highly specialized and dependent on micro-machining, their opto-mechanical interfaces (lenses, prisms, buttons, slitlets) called for separate procurement. The plan consisted hence in supplying these parts to industry for them to assemble the cable structure together with the button and slitlet units. An exception was made for the LIFU head since totaling 609 fibers in a close-packed hexagonal IFU with fore-optics and its own guide camera it represented a very challenging sub-system in itself and was entrusted to NOVA, but the cable structure was also assembled by industry.

Such an endeavor with a multiplicity of partners for the design, procurement and fabrication depends critically on the continuity of the management because of the importance of experience, informal information and quality of the communication between the stakeholders. Over the 10 years of WEAVE's development there has unfortunately been changes in the teams which added complexity: the fiber work-package manager changed twice, the director of GEPI once, the systems engineer once and SEDI-ATI was acquired by an active holding company in 2016.

\section{PROCUREMENT}

The fiber system relies on three classes of components: fiber optics including ferrules and ducts, optics with micro-optics allowing injection of light in the fibers and mechanics notably the buttons and slitlets. Procurement of the former was entrusted to industry after joint design with SEDI-ATI and lifetime testing of materials and design in the telescope environment via a MOS cable prototype.

\subsection{Optics}

The MOS prism, because of its size (1.5 mm sides), the need for 1920 prisms (plus spares) and the fact that combining the reflective and beam-forming functions into one component made it a non-standard optic, were identified as a critical component for the feasibility of the MOS subsystem in the early phases of the project when design and prototyping was on-going. The procurement risk was however clearly underestimated and it was only by 2016 that 300 such prisms were procured from BernOptics, after the FDR. Initial tolerances on the parts were based on insufficient system analysis as regards focusing the image on the tip of the fiber versus focal length of the lens, size of the prism and wavelength but also concerning alignment procedures. They were driven by the mechanical alignment of the prism to the fiber proposed by SEDI-ATI which set a $10 \mu \mathrm{m}$ tolerance on the distance between the vertex of the lens and two sides of the prism used as reference. In spite of the tightness of this tolerance, only $4.6 \%$ of these prisms failed that test and that strategy might have proved an adequate one, although costly and over-specified, if BernOptics had been able to deliver the required number of prisms within the constraints of our procurement schedule.

The cost of a large batch of such prisms was such that GEPI could only have procured them via a call for tender involving administrative documents in French, as is the law, BernOptics as a US company experienced difficulties in proceeding that way, hence procurement of more prisms was entrusted to SEDI-ATI. This administrative difficulty having been solved, it was not however before 2017 when a request for an additional 1850 prisms at the rate of 300 per month was formulated due in part to availability of funding and to technical delays. Unfortunately, more than one year after the first batch, BernOptics answered they were not available for such a job and recommended finding another supplier. 
Then began a two-year scramble for procuring these parts. No less than 29 micro-optics suppliers around the globe were contacted and a sample was taken to optical fairs to show the reality of the part which seems easy to make on the drawing due to the scaled representation. Major players declined mostly related to the size of the part but test samples were procured from Advanced Microoptic Systems GMBH, Chance4Change, Impex HighTech GMBH and Sinoptix, all well below expectations. After we had provided metrology feedback on the parts, Sinoptix produced a second test batch gratuitously and was able to deliver 228 parts by October 2018, after almost one year of collaborative efforts.

In the process of inspecting these test parts with non-contact metrology means at first remotely at the Observatoire de Versailles Saint-Quentin-en-Yvelines and finally in GEPI's workshop, the specification for the prism was fully reconsidered and a procedure for optically aligning the prism versus the back-illuminated fiber (position versus lens, tip, tilt) was designed at GEPI to make the assembly more flexible and relax tolerances. This led to the realization of an optical gluing bench in the course of 2018. This bench made Sinoptix's parts usable although they failed to meet the initial specification due to lens offsets. It was lent to SEDI-ATI and their technician was trained to its use so optical alignment eventually replaced their mechanical procedure.

This set the way forward but the administrative delay and the time required for Sinoptix to move to mass production meant the next batch of prisms did not reach us before June 2019. In the mean time, 400 prisms were ordered by the project from BernOptics, who happened to be partly available then, and received between January and April 2019. This allowed SEDI-ATI to proceed with the assembly, however, the significantly increased unit cost and limited availability of BernOptics forbade procuring more from them.
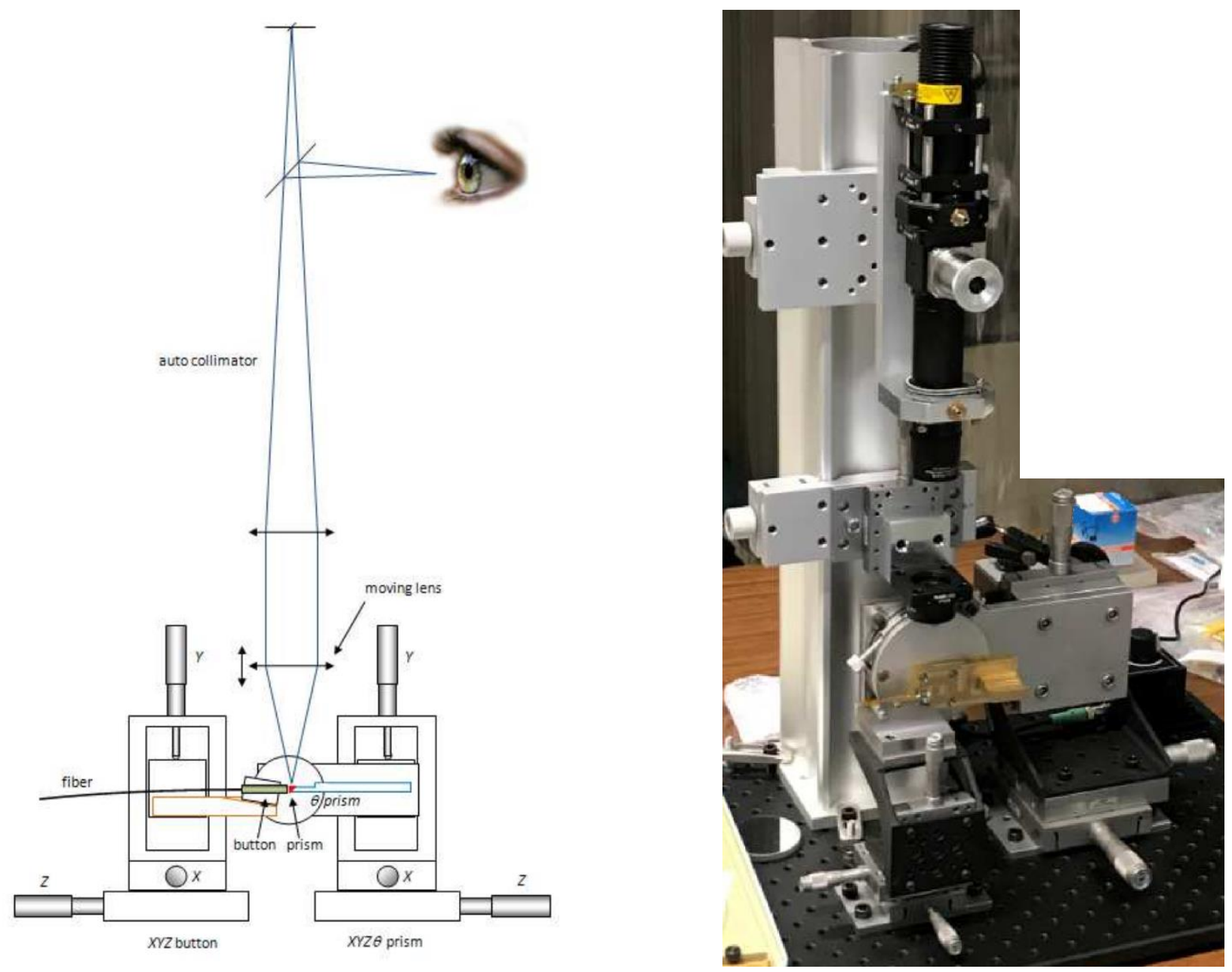

Figure 2. Schematic (left) and final version (right) of the gluing bench. 
The LIFU and mIFU optics were procured by NOVA who had carried out the corresponding optical design. With the good image quality provided by the corrector and the larger LIFU fibers and field size, the requirements on the $50 \mathrm{~mm}$ optics were not so stringent and the procurement of the two lenses was standard. For the mIFU, the separation of the lens from the prism, their slightly larger sizes $(2 \mathrm{~mm})$ and reduced numbers matched the micro-optics suppliers manufacturing abilities. They were successfully procured from Zünd Optics, first as a batch of 25 parts (for 20 needed) then, as the number of spares ran low, as an additional 10 which were all the supplier had left from fabricating the first batch.

The 25 first lenses and prisms were measured to assess conformance. Optically speaking, one lens was found to be out of specification regarding the radius of curvature of one of the dioptres (14.5 mm measured versus $14.9 \pm 0.1 \mathrm{~mm}$ required) and rejected, while all 25 prisms were within specification as concerns the $92.0^{\circ} \pm 0.5^{\circ}$ angle. Geometrically-speaking however, the initially-specified $10 \mu \mathrm{m}$ tolerance relative to the diameter of the lens and side of the prism would have caused $60 \%$ of the lenses and $28 \%$ of the prisms to be rejected making their procurement as problematic as that of the MOS prisms. Fortunately, modifying the gluing bench and procedure developed for aligning the MOS prisms it was possible to align these parts in spite of their geometric defects and increase the tolerance in a way that made all of these parts usable. As a consequence, for lack of time and based on the usability statistics for these parts, the spares which were largely used in assembling the mIFU cables were not precisely measured.

\subsection{Mechanics}

The MOS and mIFU buttons and MOS, mIFU and LIFU slitlets were high-precision mechanics because the instrument concept did not offer ways of accommodating all the geometrical defects leading to optical misalignment of the fibers ${ }^{5}$, even in the frame of the optical alignment realized using the gluing bench. They were high precision mechanics needed in large numbers.

Buttons having been designed by the positioner team were fabricated by them. Production of the buttons themselves required access to the EDM machine but, in additional to this, magnets had to be inserted and glued in the corresponding slots. Beside the geometry of the part itself, this assembly had to be carried out with great accuracy to ensure the magnet came close to the focal plate while letting the button provide the contact surface in order to control the slant precisely (within $0.1^{\circ}$ ) and did not interfere with mounting the prism at a distance of $1 \mathrm{~mm} \pm 0.5 \mathrm{~mm}$ from the front side of the button. The smallness of the part and the strength of the magnet did not make this trivial and during inspection at GEPI, a significant number of buttons were rejected and sent back to be reassembled.

One disadvantage of handling such small parts very carefully was that it was some time before the inappropriateness of the glue used became fully apparent. Due to the small size and tight fit of the slots for the magnets curing problems occurred randomly but as the magnets stuck to the buttons both magnetically and because of friction it was only when straining this bond that we realized a minority of magnets were actually loose, a defect possibly leading to catastrophic consequences on the telescope as the magnets might remain attached to the plate causing the buttons to fall off when tumbling and representing an obstacle to further fiber positioning in this region of the plate.

We subcontracted the manufacture of the slitlets to IDMM for a total of more than 150 parts with a full spare set for the $\mathrm{mIFU}$ and test parts, distributed among 3 geometries with linear tolerances down to $10 \mu \mathrm{m}$ and angular tolerances down to $0.025^{\circ}$. IDMM (and other workshops) were unwilling to machine the v-grooves holding the fibers given the even higher precision required for these both linearly and angularly. An attempt was made to machine them with the EDM machine in the UK but the $100 \mu \mathrm{m}$ produced u-grooves so the v-grooves were machined at GEPI in a second pass. Machining such a large number of structures on so many parts with different geometries and at such precisions proved a challenge. For a few parts, this would be relevant to R\&D for which our workshop is sufficiently well equipped but the repeatability and cadence required at this scale is pushing the limits of what laboratory equipment and organization is planned for. These parts were carefully measured using the non-contact metrology machines ${ }^{6}$ as the v-grooves proved excessively fragile which allowed us to verify that the added v-grooves were within tolerances.

\footnotetext{
${ }^{5}$ Typically, the slant of MOS buttons, the position of the lens in mIFU buttons, pointing variations of the fibres in the slitlets.

${ }^{6}$ The same as used to measure the prisms.
} 
This inspection also showed that the final dimensions of the parts were not always within tolerance, particularly in terms of width of the side to be polished after insertion of the fibers. With a gap between fibers in adjacent slitlets of the size of one fiber, the spacing between slitlets was very limited and this raised concerns as to whether the slits could be assembled in the end. It was the purpose of the spare mIFU slit to provide test parts allowing NOVA to develop a process for this but this was never actually carried out to full scale due to time and manpower constraints. Experience has since shown that this did not represent a real problem and the slitlets with their defects (geometry, excess of glue on side) were successfully assembled, so again a finer analysis of tolerances would have been beneficial.

\section{CABLE ASSEMBLY}

\subsection{Change of paradigm}

The assembly of the different cables was clearly the heart of the challenge. We have had a chance at GEPI, beside our experience with GIRAFFE, to experiment with all aspects of this work at a considerably reduced and simplified scale when making the MAIT cable in 2019 (a test system provided to the spectrograph for adjusting its optics and testing the slit exchange mechanism after assembly). This cable was only a few meters long, with 15 fibers distributed at the extremities and center of the mIFU slit ${ }^{7}$ and a short section of sheathing near the slitlets but it taught us a great deal about the handling of the fibers and the intricacies of the assembly. Although only needed late in the project by the spectrograph, it might have been made earlier to better understand what is at stake with assembly.

This work was delicate as fibers are intrinsically fragile and bending, twisting or exerting pressure on them increases intrinsic FRD which degrades the coupling with the spectrograph. The assembly was complex due to the installation of optical interfaces at both ends and the large number of sections relying on different materials and grouping fibers in varying numbers. These are already two properties which are hardly compatible with large volumes but, in addition, the work was also diverse because of the three cable geometries, the two button types, the three kinds of slitlets and the two different fibers used. These items seemed sufficiently akin to allow for efficiently transferring knowledge gained on one to another and that idea subtended the initial idea of having a single contractor in charge of all the assembly. However pitfalls proved numerous and forced us to change strategy.

The first and the major obstacle to this scheme proved to be the amount of work required. In 2017, SEDI-ATI had forecast the delivery of the last MOS cable for March 2019, the LIFU for August 2018, the last mIFU for January 2019 and, after the appropriate calls for tender, all contracts had been signed by the end of the year. In June 2018 however, this plan was very significantly altered as SEDI-ATI announced the delivery of the $20^{\text {th }}$ MOS shifting to February 2020 and had not started work on the LIFU nor on the mIFU. At that point in time, we had a history of being late for providing parts for prototypes and SEDI-ATI was aware of the procurement difficulties related to the MOS prisms. However, enough were available for the assembly of more than two cables, while buttons and slitlets were available for significantly more, although not yet for all. This did not coincide with SEDI-ATI's initial request have all parts prior to commencing but they had later agreed to proceed cable by cable, meaning only require having all parts for a cable prior to assembling it. The delivery of the first cable had been planned for January 2018 after all parts had been delivered in November 2017 but happened only in July 2018 and this delay was explained by personnel problems and the wish to ensure MOS cables entered production mode prior to diverting forces to the other two types of cable - although SEDIATI had carried out some pre-production in 2017 when assembling a prototype MOS cable featuring one complete unit of 24 fibers with shortened length.

Such a schedule being incompatible with WEAVE's, as the fiber links were already on the critical path prior to that announcement, it was decided that recourse to other contractors was imperative to parallelize the assembly work, in spite of the risk corresponding to entrusting such work to people whose work had not previously been evaluated, of the delay associated with new calls for tender, of the cost increase associated to replicating non-engineering costs and of the extra management required. It was agreed with SEDI-ATI that the existing contracts would be modified for them to relinquish work on the mIFU to focus on MOS cables. They insisted, however, that they could have another team assemble the LIFU within 3 months - a competitive option compared to the 2 months required by a call for tender. MOS cables being clearly the limiting item for completing the fiber system within schedule, they accepted to relinquish exclusivity on the

${ }^{7} 5$ fibres at the center and extremities of each of the central and edge slitlets. 
assembly of such cables so that another party could assemble some with the intent to fix the number of cables they would assemble later, depending on progress, in order to shorten the time to completion.

Up to 2017 relationships with SEDI-ATI had been rather informal, with limited documentation, and if the contracts provided an administrative foundation they were only poorly binding in terms of the description of the products and schedule. With a change of project manager and the uncertainty related to depending on new partners, the philosophy changed. A detailed technical specification for the MOS and mIFU cables was produced based on revisited system engineering with an exhaustive list of acceptance criteria in order to both transfer knowledge concerning the deliverables and set a foundation for future discussions with the contractors. The tasks were better defined, notably in what concerned optical alignment of the optics for which support was proposed in transferring the design, procedure and know-how related to the gluing bench developed at GEPI. Two different contractual commitments were defined for MOS and mIFU based on the specificities of each. For the former, the contract specified a quantity based on successive orders with a firm order for one and an a priori quantity of 6 cables. A clause likely to increase the overall cost of the operation as the contractor, not knowing how many cables he would assemble in the end, would not procure all supplies at once and charge the cost of the non-recurrent engineering to ensure minimum benefits even in the case of making few cables. Conversely, it offered a lever for motivating this contractor in terms of quality and schedule and offered the flexibility needed to adjust the volumes assembled by the two MOS contractors to compress the schedule. For mIFU, where fibers are many but repetition is much reduced, the non-recurrent engineering seemed key and we chose to request a simplified prototype, which was also needed for positioner work, whose evaluation would trigger the order of the 5 nominal cables. Finally, emphasis was placed on schedule.

Extensive sourcing was carried out and 26 companies contacted for pre-tender discussions. Based on recent experience, we were careful to select contractors with experience in assembling custom cables but also possessing expertise in optical engineering. Surprising as it may seem, optical fiber companies often have limited optical skills and equipment and this was a risk we were not willing to accept given the optical interfaces present in the MOS and mIFU designs. Preference was also given to companies having their own workshop, as subcontracting mechanics is susceptible of causing delays particularly during the non-recurrent phase which calls for agility when the schedule is tight. Both of these pointed towards larger companies which, additionally, might have more resources in terms of space and manpower. Indeed, in spite of the large number of fibers, the complexity of the designs and the limited total number of cables prevented a fully industrialized approach so that the effort retained a significant part of craftsmanship, in its noble sense, pushed to the limits in terms of volume. Being so highly demanding resource-wise and to avoid saturating resources, we deliberately chose to select two different contractors for MOS and mIFU, the first being FiberTech Optica (Canada) and the second a joint effort of AMS Technologies (France) and Fiberguide Industries (USA), where most of the technical work took place.

\subsection{Communication}

Scientists know there is a significant amount of risk in undertakings like WEAVE but industry is more risk-averse. For both worlds to collaborate on projects pushing technical limits, there needs to be a common understanding of what the target and boundary conditions are. Although backed by contracts, science's expectation is to have industrial partners employ all possible means of achieving the desired results within reasonable limits which is why penalties are very seldom applied. This, however, requires open and frequent communication between the parties and a fair exchange of information, sometimes as regards trade secrets knowing that they will not be disclosed.

This applies at the technical level so both parties understand what is at stake and common solutions can be found to issues. Two examples of this are worth mentioning in our case.

The first concerns the presence of dead fibers ${ }^{8}$, a criterion motivating the rejection of a cable according to all contracts and possibly leading to returning the cable to the contractor to be fixed, which in most cases would mean largely disassembling the sheathing and part of the optical interfaces to replace the faulty fibers. From the contractors' point of view this implied a considerable amount of work and additional cost, without the guarantee that the resulting cable will behave better since there is a non-negligible probability of damaging the fibers further during disassembly and

\footnotetext{
${ }^{8}$ Dead fibers: fibers with a throughput lower than $70 \%$ of the average transmission of fibers in the cable.
} 
reassembly. From WEAVE's point of view, beside the improved performance it meant significant delays in a context already driven by schedule. The case occurred several times with MOS cables from SEDI-ATI where dead fibers were identified in the course of verification, without prior information having been communicated by the contractor. Numbers remained small compared to the 960 fibers present on a plate and, time pressing, cables were accepted. It occurred a couple of times with mIFU cables from Fiberguide Industries but, this time, information was provided by the contractor prior to delivery and next steps were discussed openly. After assessing the criticality of the non-conformance within the project, central fibers being essential in the IFU while peripheric ones sample the sky background and their number only affects the precision of the corresponding estimate, additional characterization was requested and, the contractor having reported the fibers being on the edges, the cables were accepted.

The second was related to low overall throughput for all fibers of a given slitlet on one of FiberTech Optica's cable. This was only discovered during testing at GEPI as part of the photometric evaluation of the cable. With the cable in Meudon, the situation was discussed with the contractor and diagnostics carried out to identify that the problem lay in the assembly of the slitlet. Based on the shared hypothesis that re-terminating these fibers would likely solve the problem, the cable was returned to the contractor who, upon freeing the slitlet, found that stress induced when pulling the fiber in the sheathing was a first contributor to the degradation of throughput and pressure exerted on the fibers by the slitlet's cover was another. In one week's time, with frequent updates, the cable was fixed when it was highly critical that it should be returned to us quickly and final testing at GEPI showed it to be above average.

It also applies at the programmatic level, something of particular importance to meet the schedule. Visibility as to difficulties faced or progress made by SEDI-ATI has throughout been limited because communication was limited. The 6-month delay for the first two cables was never fully understood and the situation worsened with the shortage of prisms as it completely blocked assembly activities for a duration much longer than the shortage itself. It cannot be denied that the corresponding uncertainty disrupted the organization of SEDI-ATI as, understandingly, one does not wish be interrupted in the midst of such work for technical reasons but also because of personnel affectation. In the same period of time, SEDI-ATI worked on the LIFU cable for which the contract had been signed in 2017 and all parts had been delivered when requested in July 2018 but which was only delivered in June 2019.

Both FiberTech Optica and Fiberguide Industries delivered their first cables late compared to their respective schedules. However, biweekly teleconferences provided insight on the progress made and difficulties faced and with our own experience we were able to assist them. More recently, Fiberguide Industries was able to complete the last mIFU cable during the first Covid-19 shutdown and AMS technologies delivered it once Observatoire de Paris reopened. When the project management decided that, to mitigate the risk of a second lockdown, all WEAVE subsystems needed to reach the Canary Islands in September 2020, we turned to FiberTech Optica in early June to ask them to deliver the remaining 3 cables, together with fixing the defective slitlet, by end of July instead of end of August. This amounted to increasing the assembly rate by a significant factor on a short notice. They shared a Gantt diagram of their tasks, we discussed possible optimizations to their organization and they made it.

\subsection{Motivation}

A key to success is motivation. While SEDI-ATI had good records with GIRAFFE and more recently MEGARA, their motivation for the WEAVE project seems to have dwindled away. Technical and programmatic difficulties, notably the interruption in the supply chain of prisms, can explain some of it together with involvement in other projects. FiberTech Optica showed a strong motivation for the WEAVE project. When the Sinoptix prisms became available, although usable geometrically and optically speaking, they suffered from serious cleanliness issues which Sinoptix did not solve. While FiberTech Optica developed a process for cleaning them which allowed them to proceed, SEDI-ATI refused them which led to suspending activities. This was a rightful decision but is eloquent as to motivation. Fortunately, the BernOptics prisms which came later allowed them to resume assembly but with a few hand-picked clean Sinoptix prisms their total implication was reduced from a foreseen 14 to 10 cables. Conversely, FiberTech Optica was rewarded by the making of 10 cables compared to the 6 envisaged.

Fiberguide Industries also showed a strong motivation to satisfy the project. Beside the way they openly addressed the presence of dead fibers, the arrangement of the fiber array in the mIFU prototype proved insufficiently regular to 
properly sample the targets as the science desired a $5 \mu \mathrm{m}$ tolerance on the position of the fibers in the IFU. In spite of the fact that this was not specified in the contract, Fiberguide Industries revisited their process until this was met.

As a general note applicable to the three contractors and probably beyond, it is worth mentioning that cable assembly being the core of the work other aspects also calling for attention were treated as secondary. We were careful to include them among the deliverables in the later contracts to place emphasis on them and obtain satisfaction. It was the case with the shipping containers which must allow for packaging and transporting this fragile material safely and which was specified to be reusable. It was also the case for documentation in the form of a traceability matrix for the parts supplied and inspected by GEPI, assembly history and length measurements. SEDI-ATI's documentation proved only minimal with measurements which did not match with our own inspection results, while Fiberguide Industries's and FiberTech Optica's were satisfactory but difficult to obtain.

\section{VERIFICATION}

Verification is the basis for acceptance and was motivated by the technical risks. However, this characterization was also intended to support debugging and then optimizing the integration and operation of the system as a whole. Finally, it was an opportunity for a detailed look at how fiber systems perform and, analyzing results and comparing them to expectations, for science. The craftsmanship involved in the realization of the cables and the numbers were causes for significant variability among the cables and fibers. Detailed verification of the first cables indeed showed that, although the contractors learned as more cables were produced, quality did not improve significantly to then remain stable high above the conformance threshold, thereby reducing the need for verification. This led to defining an ambitious strategy targeting exhaustiveness. Verification was based on inspection and testing.

Inspection consisted in observing the cable as a whole but also the details of the optical assemblies. An inspection bench was set up in a dedicated room large enough to allow for unpacking the cables completely and safely. A detailed procedure and this bench were developed to allow for systematically assessing quality, notably sealing of the tubes and the absence of anything sharp susceptible of damaging the tubing and the fibers in the long run, as well as comparing the geometry of the cable to the specification. Given the number of sections and tubes a pragmatic approach was adopted by systematizing the installation of the cable on the bench and establishing reference markings corresponding to nominal lengths and tolerance limits. All lengths within tolerance were then easily ticked off and only those exceeding them were measured. Microscopic inspection was carried out on the test bench whose cameras provided the required high magnification. This was carried out in the flow of the test procedure to save time and reduce the need to manipulate the cables.

An optical and automated test bench was developed as reported previously ${ }^{[7,8]}$ and it proved capable of automating the tests via an ad hoc script system. The scope of the tests was extended to match the acceptance criteria of the later contracts by measuring throughput and FRD in 6 color bands and white light, as well as the orientation of the output beam. As the bench had not been planned for photometric measurements, we relied on relative photometry by comparing MOS fibers' throughput to that of a short reference fiber of the same type fixed on the bench and applying corrections based on a differential model of fibers' properties. Much work was required to automate the procedure and make it robust to changes in the hardware being tested but $100 \%$ reliability was never achieved. From time to time posterior manual testing allowed confirming which of the fiber and the testing was failing and achieve trustworthy results in the latter case. Iterative centering to inject light in the fiber was carried out in a way similar to what will happen on the telescope and differences in the polishing of the MOS prisms ${ }^{9}$ and variability in the button assemblies caused issues which were only gradually solved. Often these issues were associated to focusing, something which for better or for worse will not happen for each fiber individually on the telescope.

\footnotetext{
${ }^{9}$ The edge of the lensed side was completely polished for Sinoptix but not for BernOptics.
} 

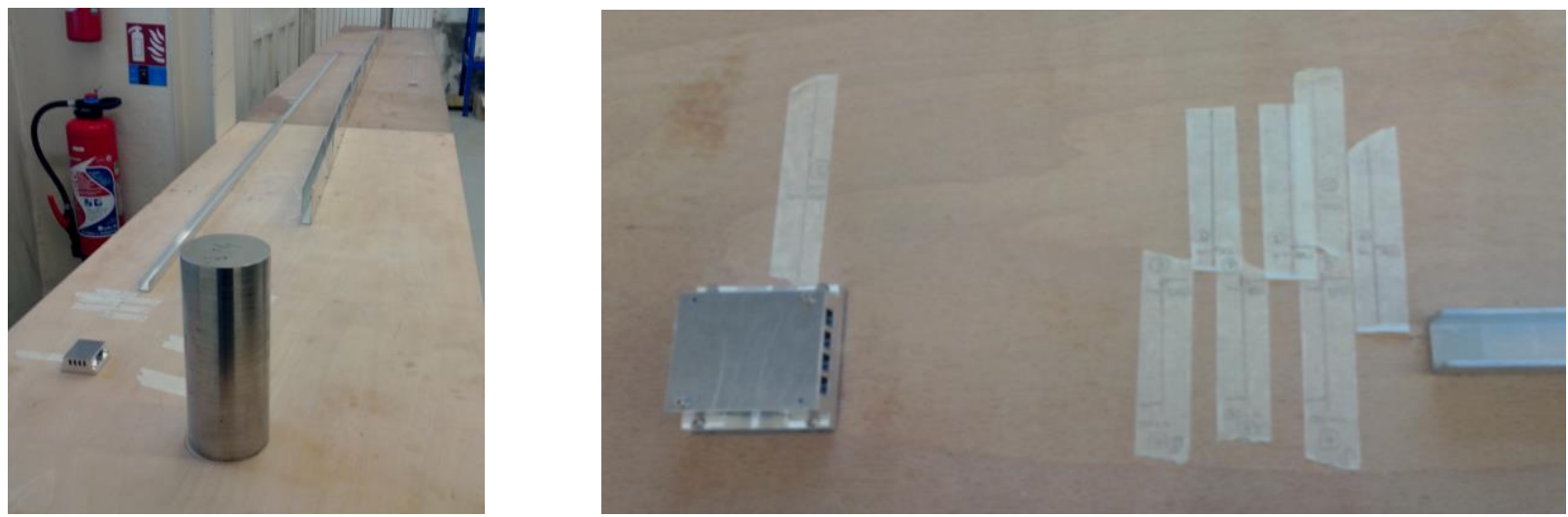

Figure 3. 6-m long inspection bench with structures used to position cables in a repeatable way (left), detail of the mechanical block used to install slitlet with nominal and maximum tolerance markings (right).
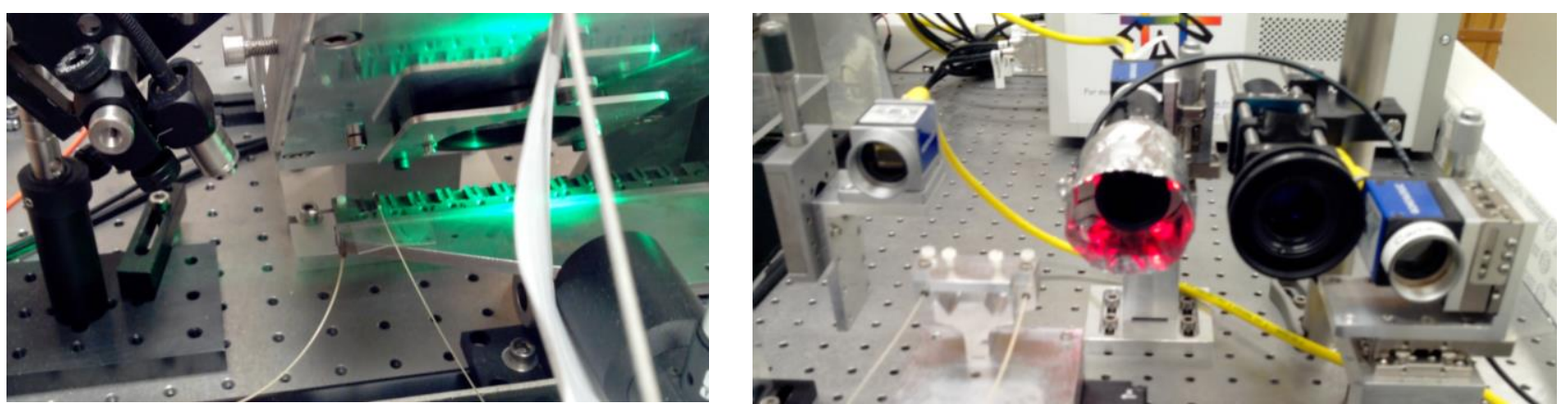

Figure 4. Button side of the test bench with the reference fibre (white ferrule) and a MOS button installed and midway between the objective allowing for injecting a collimated beam in the fibre for the ring test and the output of the telescope simulator (left) and slitlet side with the ring and near field cameras, back-illumination source and far-field camera from left to right (right).

The bench's limited capability in terms of buttons simultaneously installed required testing MOS cable in four passes. This installation being manual and the need to give test results a quick look prior to exchanging buttons proved a management headache as it called for high availability of personnel trained to manipulate the fibers, look at the results and manipulate the test software. With a test sequence lasting 6.5 hours it proved difficult to test more than 24 fibers a day which led to one cable tested per week on average. When pressure to ship the cables for integration to the positioner became maximum, this called for week-end interventions and reducing the number of bands used to shorten testing for the last few MOS cables to increase the cadence. Integration to the positioner has been a long and delicate task involving manipulating the fibers a lot. As a consequence, as for the assembly work by industry, every possible effort was made to ensure the supply chain would not be interrupted and it has been the project's policy to compress testing to meet the positioner's demand. In spite of this, all cables but 1.5 MOS cables were tested - missing tests being the result of the failure of the computer controlling the bench and the time required to replace it.

Inspection and testing proved a significant effort that had been largely underestimated in terms of manpower and so put significant strain on the team. All cables except the LIFU were inspected. Multiple non-conformance relative to section lengths on MOS cables from SEDI-ATI and FiberTech Optica were uncovered or confirmed. After discussing the corresponding integration risks with the interfacing systems, these cables were nevertheless accepted. Three cables were returned to SEDI-ATI for fixes related to sealing of the conduits, polishing of three slitlets and realignment of a MOS prism. As mentioned in section 5.2, testing allowed for identifying dead fibers and a problematic slitlet which led to one cable being returned to FiberTech Optica. The combination of inspection and testing showed that although the large number of fibers provides some amount of redundancy, cables couples fibers in such a way that a defect can affect multiple fibers. Although contractors improved their processes over time our exhaustive verification strategy was confirmed by finding issues even among the last cables. 
The LIFU cable was neither inspected nor tested prior to being shipped to NOVA for the assembly of the fiber head. Inspection was not carried out because the contract failed to specify a reusable shipping container and, because of the late delivery, the pressure for shipping LIFU was such that there was no time to extract, inspect and repackage it. The fact that the 609 fibers at the telescope's end were bare and not polished also made inspection risky and forbade any testing. Since then the head was successfully installed and the slit assembled but this subsystem has still not been tested finely, which represents a risk for the project.
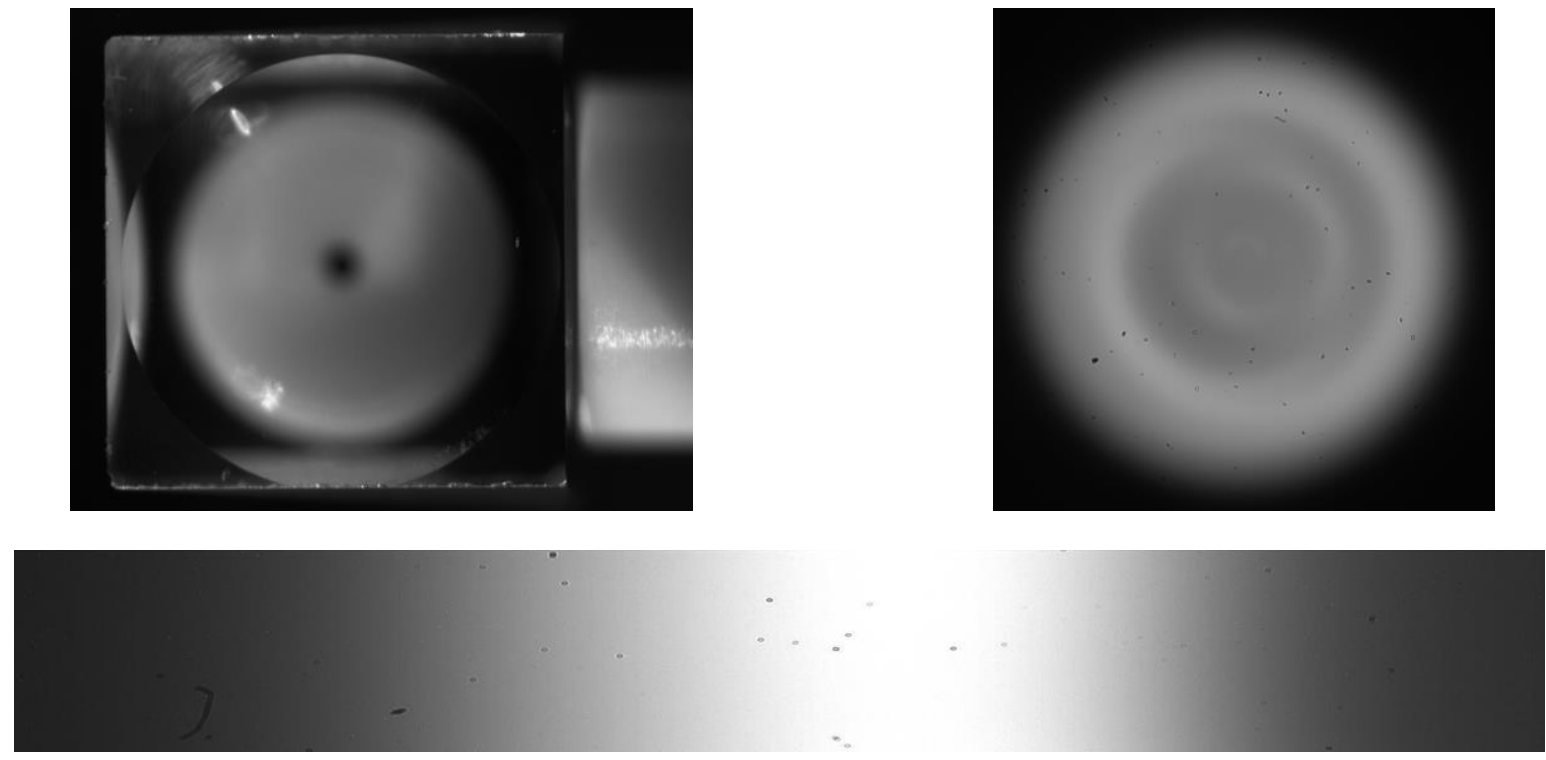

Figure 5. Sample of images acquired with the test bench: close-up on a Sinoptix prism whose lens is decentered with a fibre properly aligned to its optical axis (top left), white far-field image acquired to measure FRD and relative photometry (top right), left portion of ring obtained by injecting a collimated beam at an angle to measure the pointing of the fibre (bottom).

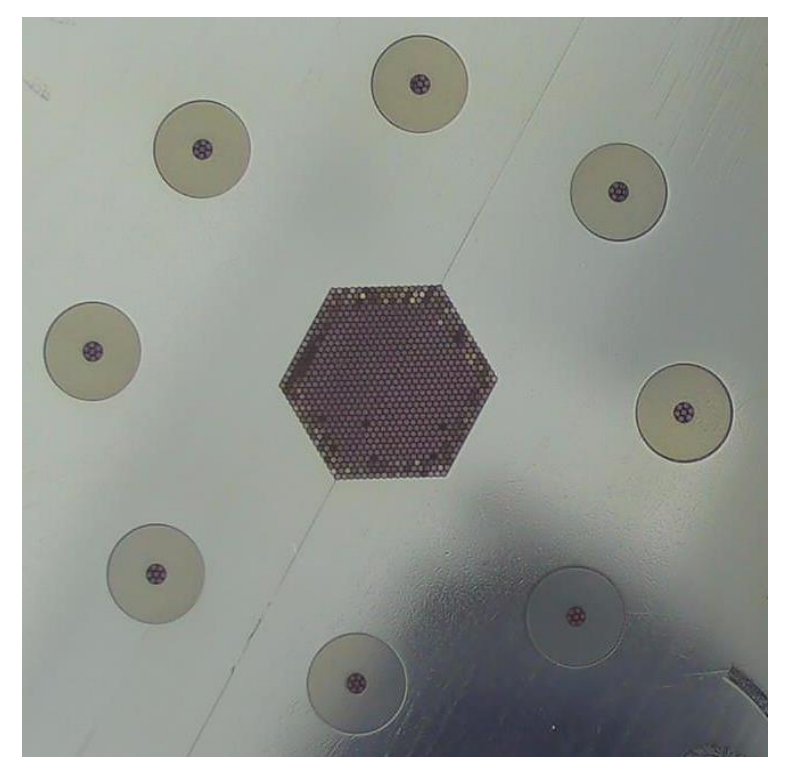

Figure 6. Assembled LIFU fibre head with its large 547-fiber central IFU (with 3 outer rings of fiber protecting the science fibers) and 8 7-fiber IFUs distributed on the periphery (2.5 arcminutes away from the center) and dedicated to measuring the sky. 


\section{INTEGRATION}

Integration of the fibers to the instrument is threefold. On the positioner's end, guide, MOS and mIFU fibers needed to be inserted in the retractors, which made it possible to configure the positioner finely and carry out more realistic tests than were done with the MOS and mIFU prototypes, while the LIFU head needed to be mounted on the structure. All fibers were then routed out of the positioner and placed in crates. On the spectrograph's end, the slitlets needed to be assembled on the slit mounts, cleaned, immersed in optical gel then pushed against the field lens. This was done at NOVA for the LIFU but in the UK for the other three slits. Final integration of these slits in the spectrograph will only happen at the telescope although tests were carried out with the LIFU slit. Once the positioner is installed on the telescope, the fibers will also needed to be routed along the telescope structure. As WEAVE is planned to be taken off the telescope periodically, though not often, this interface has been designed to be easier to manage and tests have already been carried out using empty conduits as a substitute for the fibers.

Adding the mIFU buttons on plate B impacted accommodating all 960 MOS fibers because the volume around the focal plate limited the installation of additional retractors. By design, only 940 MOS fibers are deployable on this plate, however we chose to make MOS-B identical to MOS-A and the 20 buttons which cannot be deployed are stored as spares within the retractors. This decision prevented further increasing diversity in the system and made MOS A and MOS B cables interchangeable - as a matter of fact cables labelled MOS A ended up being installed on plate B and viceversa, except for cable 10, as a result of the chronological sequence of deliveries and the schedule of the integration in the positioner. The 20 spare fibers also offered an opportunity to optimize the performance at first light by discriminating against fibers of lower quality. This was limited by the fact that the schedule led cables to be integrated in the same order as they were received by GEPI and by the distribution of retractors hosting these fibers. Verification results provided live guidance to integration in this respect.

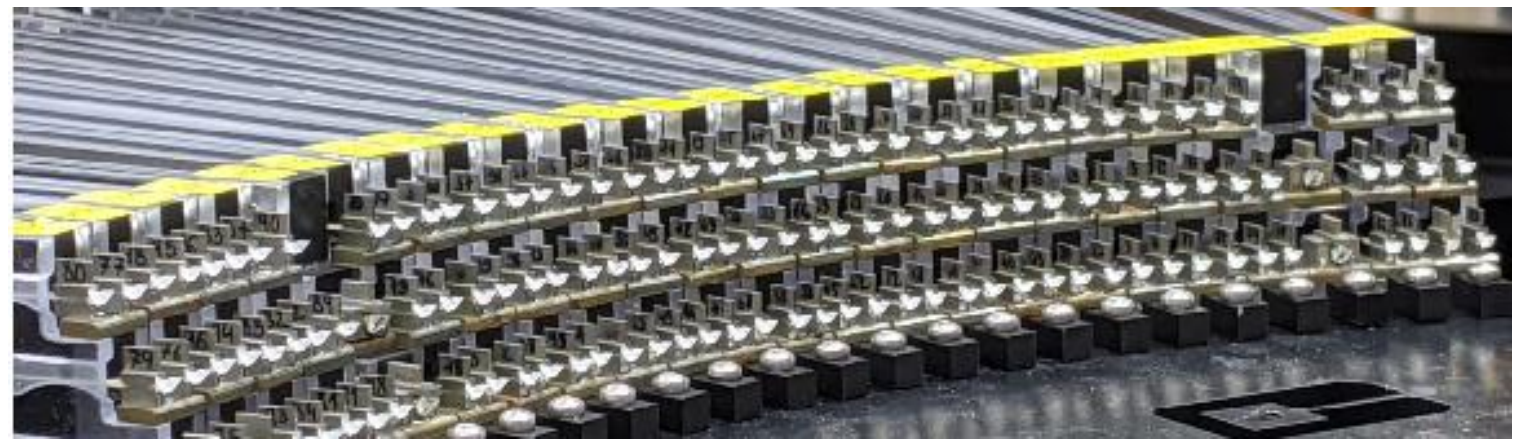

Figure 7. MOS, mIFU guide buttons positioned on the retractors at the edge of plate B.

\section{CONCLUSIONS}

All 3285 fibers pertaining to the four fiber systems have now been integrated to the positioner with only a few dead fibers and a few missing MOS prisms knocked off during integration and testing of the positioner. A gluing bench has been delivered with spare parts to replace these prisms and carry out maintenance over the course of WEAVE's life. Fiber-related activities are coming to an end with efforts now on publishing the as-built documentation and final analysis of all the test data. Concerning the instrument, the interested reader is advised to turn the paper ${ }^{[5]}$ describing the overall status of WEAVE in these proceedings.

We have learned many a lesson over the years. One is certainly that interfaces are key. Key in the way they are specified - sometimes missing or over-specified - but also in determining what the implications are of failing to meet tolerances to serve as guidance when difficult management decisions must be made as regards acceptance when pressure increases. 
Key also in considering who will fabricate and test them, as our experience has shown that the fiber optics industry in general has limited expertise in optics ${ }^{10}$.

Another is that fiber systems of such size and complexity require expertise from laboratories and industry and that the assembly stage is challenging. This challenge is best tackled when the two collaborate, which implies open and frequent discussion and mutual acceptation of risks. This extends beyond a contractual relationship and requires industry to be highly motivated. The early stages of a project are a favorable moment to develop such relationships, via co-design and prototyping, while pressure is still low but efforts will be needed to preserve them until the end. Yet single-point failures must be avoided, in particular depending on a single provider or industrial partner, so sourcing early in the project needs to identify alternatives.

Long-term preparation of verification is also recommended. Beside allowing for fully exploiting prototypes, it allows for a sounder knowledge of what is at stake so that when the deliverables arrive there remains fewer uncertainties associated to the testing itself. It favors carrying out verification in a timely manner, contractually and to provide feedback to industry, without excessive stress and or causing delays.

Finally, technical and programmatic aspects are challenging enough not to add additional management difficulties. GEPI's and OCA's ${ }^{11}$ involvement allowed the French astronomical community to join WEAVE, which means contributing to the preparation of the Galactic Archeology survey and future access to the data while France is not a contributor to the William Herschel Telescope. As the first ground spectroscopic follow-up of Gaia in which France is a major partner this is highly strategic scientifically-speaking. Yet support has been fragmentary so manpower was scarce and funds were collected bit-by-bit over the duration of the project from no less than 5 different sources, each with its own rules, thereby considerably complicating administration and reducing the project's ability to move fast when required ${ }^{12}$.

\section{ACKNOWLEDGEMENTS}

We wish to thank all our industrial partners for their contributions in making the fiber system a reality, IDMM, BernOptics, Sinoptix, Zünd Optics and for the cable assembly SEDI-ATI, FiberTech Optica, AMS Technologies and Fiberguide Industies. This was also only made possible thanks to funding from Région Île-de-France through the DIM ACAV program, INSU, Observatoire de Paris, the WEAVE project and a financial contribution from Université de Franche-Comté. Finally, shipping supplies and cables across the globe has also been quite an effort and we are grateful to CNRS's Ulisse service and in particular Guillaume Bel for his constant and excellent assistance.

\section{REFERENCES}

[1] Dalton, G. et al., "WEAVE: the next generation wide-field spectroscopy facility for the William Herschel Telescope", Proc. SPIE 8446 (2012).

[2] Dalton, G. et al., "Project overview and update on WEAVE: the next generation wide-field spectroscopy facility for the William Herschel Telescope", Proc. SPIE 9147 (2014).

[3] Dalton, G. et al., "Final design and progress of WEAVE: the next generation wide-field spectroscopy facility for the William Herschel Telescope", Proc. SPIE 9908 (2016).

[4] Dalton, G. et al., "Construction progress of WEAVE: the next generation wide-field spectroscopy facility for the William Herschel Telescope", Proc. SPIE 10702 (2018).

[5] Dalton, G. et al., "Final integration and early testing of WEAVE: the next generation wide-field spectroscopy facility for the William Herschel Telescope", Proc. SPIE 11447 (2020).

${ }^{10}$ Our experience with FiberTech Optica has shown that they are more proficient than others in this respect.

${ }^{11}$ OCA contributed to the study of the field corrector in the early days of WEAVE.

${ }^{12}$ For instance for procuring prisms from BernOptics in 2016. 
[6] Guinouard, I. et al., "Development of the single fibres and IFUs of WEAVE", Proc. SPIE 8450 (2012).

[7] Sayède, F. et al., "WEAVE MOS fibre bundle test plan", Proc. SPIE 9151 (2014).

[8] Sayède, F. et al., "First results of tests on the WEAVE fibres", Proc. SPIE 9912 (2016).

[9] Schallig, E. et al., "Developments in fiber-positioning technology for the WEAVE instrument at the William Herschel Telescope", Proc. SPIE 9908 (2016).

[10] Schallig, E. et al., "First lab results of the WEAVE fibre positioner system", Proc. SPIE 10702 (2018).

[11] Hughes, S. et al., "Final assembly, metrology, and testing of the WEAVE fibre positioner", Proc. SPIE 11447 (2020).

[12] Tomàs, A. et al., "The WEAVE prime focus correction: from design to integration", Proc. SPIE 10706 (2018).

[13] Stuik, R. et al., "Integration and testing of the WEAVE Spectrograph", Proc SPIE 11447 (2020). 